\section{Metabolically Healthy Obesity: Is It Really a Benign Condition?}

\author{
Hwi Seung Kim ${ }^{1,2}$, Chang Hee Jung ${ }^{1,2, *}$ \\ 'Department of Internal Medicine, Asan Medical Center, University of Ulsan College of Medicine, Seoul; \\ ${ }^{2}$ Asan Diabetes Center, Asan Medical Center, Seoul, Korea
}

\author{
Received January 25, 2021 \\ Reviewed February 11, 202 \\ Accepted February 18, 2021 \\ ${ }^{*}$ Corresponding author \\ Chang Hee Jung \\ https://orcid.org/0000-0003-4043-2396 \\ Department of Internal Medicine, Asan \\ Medical Center, University of Ulsan \\ College of Medicine, 88 Olympic-ro \\ 43-gil, Songpa-gu, Seoul 05505, Korea \\ Tel: +82-2-3010-1418 \\ Fax: +82-2-3010-6962 \\ E-mail: chjung0204@gmail.com
}

Obesity is associated with various cardiometabolic diseases and increased mortality. ${ }^{1}$ However, a select group of obese individuals, the metabolically healthy obese ( $\mathrm{MHO}$ ), characterized by absence of metabolic disturbances, appear to be spared from the ominous complications of obesity. ${ }^{2,3}$ In South Korea, the prevalence of obesity has increased in all age groups over the last decade and reached $35.7 \%$ in $2018 .{ }^{1}$ According to a meta-analysis, the prevalence of MHO reported in previous studies was largely variable depending on the definition of $\mathrm{MHO}$ used, ranging from $13 \%$ to $86 \%$. $^{2}$ There are also regional and sex differences in the prevalence of $\mathrm{MHO}^{2}$ Although the mechanism of preservation of metabolic health in the $\mathrm{MHO}$ group has been investigated in both human and animal studies, it remains unknown. ${ }^{3,4}$ The suggested mechanisms include subcutaneous fat distribution, adipose tissue expandability, sustained insulin sensitivity, preserved adipocyte function, chronic inflammation, and genetic associations. ${ }^{3-5}$ Environmental and behavioral factors, such as an individual's physical activity level and selfrated health $(\mathrm{SRH})$, also have been proposed. ${ }^{6}$

The prognosis of $\mathrm{MHO}$ in association with type 2 diabetes and cardiovascular disease (CVD) is controversial. Some studies have demonstrated that $\mathrm{MHO}$ individuals show a favorable prognosis and advocated that $\mathrm{MHO}$ be classified as a "benign condition."5
However, some studies have opposed this concept and reported that $\mathrm{MHO}$ individuals have higher risk of type 2 diabetes, CVD, and mortality. ${ }^{7}$ Accumulated evidence regarding the increased risk of type 2 diabetes and CVD among MHO individuals compared to that in metabolically healthy normal weight (MHNW) individuals suggests that $\mathrm{MHO}$ is not a "benign condition."3,7

In a previous study, the SRH of subjects with obesity and metabolic syndrome was worse than that reported by non-obese subjects. ${ }^{6}$ In addition, low SRH in MHO individuals was associated with progression to metabolically unhealthy obese (MUO) status. ${ }^{6}$ In another study, impaired glucose tolerance was associated with low $\mathrm{SRH} .{ }^{8}$ In line with these results, a recent study by Hjelmgren et al. ${ }^{9}$ published in Journal of Obesity \& Metabolic Syndrome reported that obese individuals rated their health worse than non-obese individuals. This study reported a novel finding that $\mathrm{MHO}$ subjects rated their health similar to other individuals of the same age. Moreover, the SRH of the MUO group was significantly worse than those of the MHNW and MHO groups. These findings imply that metabolic health has a more critical influence on one's health rating than does obesity itself.

Previous studies on the level of physical activity of $\mathrm{MHO}$ individuals have reported inconsistent results. ${ }^{10,11}$ A study by de Winter 
et al. ${ }^{11}$ reported no difference in the level of physical activity between MHO and MUO groups; however, de Rooij et al. ${ }^{10}$ concluded that the $\mathrm{MHO}$ group performed more physical activity than the MUO group. A study by Hjelmgren et al. ${ }^{9}$ provided evidence supporting the study by de Winter et al. $^{11}$; the number of individuals performing leisure-time physical activity for $>30$ minutes at least 1-2 times a week was not significantly different between $\mathrm{MHO}$ and MUO groups. In addition, the level of physical activity in the MHNW group was similar to that in obese groups, possibly because the study was a cross-sectional analysis of a relatively small number of obese subjects. Therefore, it seems premature to purport a conclusion on the effect of the level of physical activity on metabolic health and body weight based on the abovementioned results alone.

However, Hjelmgren et al. ${ }^{9}$ showed that the MHO group had intermediate levels of beta-cell dysfunction (indirectly measured by plasma proinsulin and C-peptide levels) and insulin resistance assessed by homeostatic model assessment for insulin resistance. Currently, MHO subjects are considered metabolically heterogeneous in terms of risk of type 2 diabetes, although they are classified using different diagnostic criteria. ${ }^{12}$ Our group demonstrated that elevated high-sensitivity C-reactive protein level or increased hepatic steatosis could stratify the risk of type 2 diabetes among MHO subjects. ${ }^{13,14}$ Although the study by Hjelmgren et al. ${ }^{9}$ confirmed the intermediate status of $\mathrm{MHO}$ subjects in terms of betacell function and insulin resistance, the heterogenous nature of MHO should be considered when assessing the risk of type 2 diabetes in such a population.

The homogenous population of this study is both a strength and a limitation. Another limitation of this study is the cross-sectional study design, whereas metabolic health and obesity status should be considered a dynamic concept. ${ }^{15}$ Nevertheless, the present study provides valuable information on SRH, level of physical activity, and insulin resistance in MHO individuals. This study also supplements evidence that $\mathrm{MHO}$ status is not comparable to $\mathrm{MHNW}$ status, as various studies have categorized $\mathrm{MHO}$ as an intermediate phenotype between MHNW and MUO phenotypes. The debate on the MHO phenotype and its relationship with possible causes and consequences of obesity persists. Further studies are needed to provide a risk-stratified obesity treatment in $\mathrm{MHO}$ patients.

\section{CONFLICTS OF INTEREST}

Chang Hee Jung has worked as an Associate Editor of Journal of Obesity \& Metabolic Syndrome; however, he was not involved in peer reviewer selection, evaluation, or decision process of this article. No other potential conflicts of interest relevant to this article were reported.

\section{ACKNOWLEDGMENTS}

This work was supported by the Basic Science Research Program through the National Research Foundation of Korea (NRF) funded by the Ministry of Education (grant No. NRF-2020R1A2C1101977 to CHJ). This funding source had no roles in the design of the study; the collection, analysis, and interpretation of data; writing of the article; or the decision to submit the article for publication.

\section{AUTHOR CONTRIBUTIONS}

Study concept and design, acquisition of data, and analysis and interpretation of data: all authors; drafting of the manuscript: HSK; critical revision of the manuscript: $\mathrm{CHJ}$; statistical analysis: HSK; obtained funding, administrative, technical, or material support, and study supervision: $\mathrm{CHJ}$.

\section{REFERENCES}

1. Nam GE, Kim YH, Han K, Jung JH, Rhee EJ, Lee SS, et al. Obesity fact sheet in Korea, 2019: prevalence of obesity and abdominal obesity from 2009 to 2018 and social factors. J Obes Metab Syndr 2020;29:124-32.

2. Lin H, Zhang L, Zheng R, Zheng Y. The prevalence, metabolic risk and effects of lifestyle intervention for metabolically healthy obesity: a systematic review and meta-analysis: a PRIS MA-compliant article. Medicine (Baltimore) 2017;96:e8838.

3. Stefan N, Häring HU, Hu FB, Schulze MB. Metabolically healthy obesity: epidemiology, mechanisms, and clinical implications. Lancet Diabetes Endocrinol 2013;1:152-62.

4. Loos RJ, Kilpeläinen TO. Genes that make you fat, but keep 
you healthy. J Intern Med 2018;284:450-63.

5. Primeau V, Coderre L, Karelis AD, Brochu M, Lavoie ME, Messier V, et al. Characterizing the profile of obese patients who are metabolically healthy. Int J Obes (Lond) 2011;35: 971-81.

6. Kim MH, Chang Y, Jung HS, Shin H, Ryu S. Impact of selfrated health on progression to a metabolically unhealthy phenotype in metabolically healthy obese and non-obese individuals. J Clin Med 2019;8:34.

7. Kramer CK, Zinman B, Retnakaran R. Are metabolically healthy overweight and obesity benign conditions? A systematic review and meta-analysis. Ann Intern Med 2013;159:758-69.

8. Andersson S, Ekman I, Friberg F, Daka B, Lindblad U, Larsson CA. The association between self-rated health and impaired glucose tolerance in Swedish adults: a cross-sectional study. Scand J Prim Health Care 2013;31:111-8.

9. Hjelmgren O, Gummesson A, Bergström G, Schmidt C. Betacell function, self-rated health, and lifestyle habits in 64-yearold Swedish women with metabolically healthy obesity phenotype. J Obes Metab Syndr 2020;29:39-46.

10. de Rooij BH, van der Berg JD, van der Kallen CJ, Schram MT, Savelberg HH, Schaper NC, et al. Physical activity and seden- tary behavior in metabolically healthy versus unhealthy obese and non-obese individuals: the Maastricht study. PLoS One 2016;11:e0154358.

11. de Winter M, Rioux BV, Boudreau JG, Bouchard DR, Sénéchal M. Physical activity and sedentary patterns among metabolically healthy individuals living with obesity. J Diabetes Res 2018;2018:7496768.

12. Bell JA, Kivimaki M, Hamer M. Metabolically healthy obesity and risk of incident type 2 diabetes: a meta-analysis of prospective cohort studies. Obes Rev 2014;15:504-15.

13. Jung CH, Lee MJ, Kang YM, Jang JE, Leem J, Hwang JY, et al. The risk of incident type 2 diabetes in a Korean metabolically healthy obese population: the role of systemic inflammation. J Clin Endocrinol Metab 2015;100:934-41.

14. Jung CH, Kang YM, Jang JE, Hwang JY, Kim EH, Park JY, et al. Fatty liver index is a risk determinant of incident type 2 diabetes in a metabolically healthy population with obesity. Obesity (Silver Spring) 2016;24:1373-9.

15. Soriguer F, Gutiérrez-Repiso C, Rubio-Martín E, García-Fuentes E, Almaraz MC, Colomo N, et al. Metabolically healthy but obese, a matter of time? Findings from the prospective Pizarra study. J Clin Endocrinol Metab 2013;98:2318-25. 